Marianne Roth

Prise de position de l'ASP sur la stratégie de la qualité et les objectifs quadriennaux du Conseil fédéral

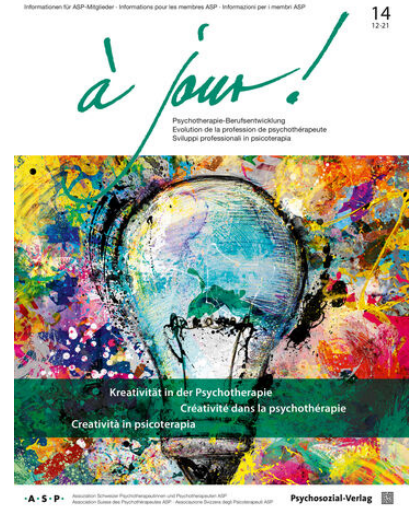

à jour!

Psychotherapie-Berufsentwicklung

7. Jahrgang, Nr. 2, 2021, Seite 63-64

DOI: $10.30820 / 2504-5199-2021-2-63$

Psychosozial-Verlag 


\section{Impressum | Mentions légales}

\author{
à jour! - Psychotherapie-Berufsentwicklung \\ ISSN 2504-5199 (Print-Version) \\ ISSN 2504-5202 (digitale Version) \\ 7. Jahrgang Heft 2/2021, Nr. 14 \\ https://doi.org/10.30820/2504-5199-2021-2
}

\section{Herausgeber}

Assoziation Schweizer Psychotherapeutinnen und Psychotherapeuten ASP

Riedtlistr. 8 | 8006 Zürich | Tel.: 0432689300 | www. psychotherapie.ch

Die Zeitschrift à jour! Psychotherapie-Berufsentwicklung ist ein Informationsorgan der ASP. Gleichzeitig versteht sie sich als Forum ihrer Mitglieder, in dem auch Meinungen geäussert werden, die unabhängig von der Meinung des Vorstandes und der Redaktion sind.

\section{Redaktion}

Peter Schulthess | Redaktionsleitung peter.schulthess@psychotherapie.ch|Tel.: 0765591920

Marianne Roth | marianne.roth@psychotherapie.ch

Veronica Defiébre | veronica.defiebre@psychotherapie.ch

Sandra Feroleto|sandra.feroleto@psychotherapie.ch

\section{Redaktionsschluss}

1. März für Juni-Heft / 15. September für Dezember-Heft

\section{Verlag}

Psychosozial-Verlag

Walltorstr. 10 | D-35390 Gießen | Tel.: +4964196997826

www.psychosozial-verlag.de |info@psychosozial-verlag.de

\section{Abo-Verwaltung | Bezugsgebühren}

Psychosozial-Verlag | bestellung@psychosozial-verlag.de

Jahresabonnement 29,90€ (zzgl. Versand)

Einzelheft 19,90€ (zzgl. Versand)

Studierende erhalten gegen Nachweis $25 \%$ Rabatt.

Das Abonnement verlängert sich um jeweils ein Jahr, sofern nicht eine Abbestellung

bis acht Wochen vor Ende des Bezugszeitrasums erfolgt.

Das Abonnement ist für ASP-Mitglieder im Mitgliedsbeitrag enthalten.

\section{Anzeigen}

Anfragen zu Anzeigen richten Sie bitte an den Verlag (anzeigen@psychosozial-verlag.de) oder die Geschäftsstelle der ASP (asp@psychotherapie.ch).

Es gelten die Preise der auf www. psychosozial-verlag.de einsehbaren Mediadaten. ASP-Mitglieder wenden sich bitte direkt an die Geschäftsstelle der ASP.

Bildnachweise

Titelbild: @ iStock by Getty Images/RapidEye (ID 912441162)

\section{Digitale Version}

Die Zeitschrift à jour! Psychotherapie-Berufsentwicklung ist auch online einsehbar: www.a-jour-asp.ch

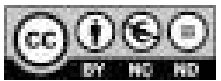

Die Beiträge dieser Zeitschrift sind unter der Creative Commons Attribution-NonCommercial-NoDerivs 3.0 DE Lizenz lizensiert. Diese Lizenz erlaubt die private Nutzung und unveränderte Weitergabe, verbietet jedoch die Bearbeitung und kommerzielle Nutzung. Weitere Informationen finden Sie unter: creativecommons.org/licenses/by-nc-nd/3.0/de

\author{
à jour! - Evolution de la profession de psychothérapeute \\ ISSN 2504-5199 (Version papier) \\ ISSN 2504-5202 (Version numérique) \\ 7. tome numéro 2/2021, 14 \\ https://doi.org/10.30820/2504-5199-2021-2
}

\section{Editeur}

Association Suisse des Psychothérapeutes ASP

Riedtlistr. 8 | 8006 Zürich | Tel.: 0432689300 | www. psychotherapie.ch

La revue à jour! Évolution de la profession du psychothérapeute est un organe d'information de l'ASP. En plus, c'est un forum dans lequel on exprime des avis qui sont indépendants de l'avis du comité et de la rédacion.

\section{Rédaction}

Peter Schulthess | Directeur de rédacion

peter.schulthess@psychotherapie.ch | Tel.: 0765591920

Marianne Roth / marianne.roth@psychotherapie.ch

Veronica Defiébre /veronica.defiebre@psychotherapie.ch

Sandra Feroleto|sandra.feroleto@psychotherapie.ch

\section{Date de rédaction finale}

1er mars pour juin | 15 septembre pour décembre

\section{L'éditeur}

Psychosozial-Verlag

Walltorstr. 10 | D-35390 Gießen | Tel.: +49641969978 26

www.psychosozial-verlag.de|info@psychosozial-verlag.de

\section{Gestion des abonnements | Frais de souscription}

Édition psychosociale | bestellung@psychosozial-verlag.de

Abonnement annuel 29,90€ (frais d'envoi en sus)

Prix du numéro 19,90€ (frais d'envoi en sus)

Les étudiants bénéficient d'une réduction de $25 \%$ sur présentation d'un justificatif.

L'abonnement est reconduit d'un an à chaque fois dans la mesure où aucune résiliation n'a lieu avant le 15 novembre.

L'abonnement est compris dans la cotisation pour les membres ASP.

\section{Annonces}

Veuillez adresser vos demandes de renseignements sur les annonces à l'éditeur (anzeigen@psychosozial-verlag.de) ou au bureau de I'ASP (asp@psychotherapie.ch). Les prix valables sont ceux publiés dans les données médiatiques sur www.psychosozial-verlag.de.

Les membres ASP sont priés de s'adresser directement à la rédaction.

\section{Crédits photographiques}

Couverture: $\odot$ iStock by Getty Images/RapidEye (ID 912441162)

\section{Version numérique}

La revue à jour! Psychothérapie-Développement professionnel est également consultable en ligne : www. a-jour-asp.ch

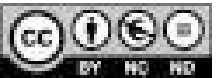

Les articles de cette revue sont disponibles sous la licence Creative Commons 3.0 DE en respectant la paternité des contenus - pas d'utilisation commerciale - sans œuvre dérivée. Cette licence autorise l'utilisation privée et la transmission sans modification, interdit cependant le traitement et l'utilisation commerciale. Veuillez trouver de plus amples informations sous : creativecommons.org/licenses/by-nc-nd/3.0/de 


\title{
Prise de position de l'ASP sur la stratégie de la qualité et les objectifs quadriennaux du Conseil fédéral
}

\author{
Marianne Roth
}

Dans le cadre d'une procédure de consultation sur la stratégie et les objectifs quadriennaux concernant la sécurisation et la promotion de la qualité des prestations que le Conseil fédéral a lancé en été, l'ASP a pu prendre position avec d'autres acteurs du domaine de la santé. Les efforts déployés par la fédération pour poursuivre le développement de la qualité des prestations au bénéfice des patientes et des patients et de les rendre plus visibles valent la peine d'être soutenus. La stratégie laisse cependant sans réponses des questions auxquelles il faut impérativement répondre.

\section{La participation et le partage des rôles doivent être clarifiés}

Un facteur de succès central pour atteindre les objectifs formulés est l'implication des fournisseurs de prestations concernés. Ce principe fondamental n'est pas assez identifiable dans la version actuelle de la stratégie de la qualité et dans les objectifs quadriennaux. La pertinence des différents fournisseurs de prestations et les activités poursuivies pendant des années pour développer les associations professionnelles doivent être prises en compte en tant que base fondamentale dans la stratégie. Une participation des associations professionnelles dans la Commission fédérale pour la qualité (CFQ) récemment créée n’est pas prévue. C'est ainsi le personnel soignant y est certes représenté en tant que plus grand groupe professionnel dans le domaine de la santé, ce que lon doit saluer, mais pas d'autres groupes professionnels directement concernés par les activités de la commission ou leurs associations.

Le partage des rôles entre la Fédération, les cantons et les acteurs du domaine de la santé doit être respecté et régler de façon plus claire. Les tâches de la fédération devraient principalement consister en la définition de bases valables pour tous les domaines et groupes professionnels et de se concentrer sur le plan macro. La révision de la LAMal exige explicitement la conclusion de contrats de qualité entre les associations des fournisseurs de prestations et les associations des assureurs. C'est la raison pour laquelle il faut accorder une liberté de négociation. Les documents interviennent cependant massivement dans le domaine de compétence des fournisseurs de prestations et des assureurs en préconisant déjà des consignes détaillées au niveau des contrats et des concepts de qualité. Les documentes n'abordent pas la problématique des fournisseurs de prestations qui n'appartiennent à aucune association professionnelle. Qui conclut les contrats avec eux ? Qui les contrôle ? Le rôle des associations professionnelles, notamment dans le domaine de la psychothérapie, où il n'y a pas d'obligation d'appartenance à une association, reste peu clair.

La stratégie de la qualité doit également clarifier les choses en ce qui concerne les exigences contradictoires du législateur. Le point suivant est par exemple peu clair : les règles s'appliquant à la protection des données pour les mesures de la qualité pour utiliser les données rapportées aux patientes et aux patients pour des mesures d'amélioration de la qualité et pour la transparence exigée. C'est uniquement lorsque les conditions-cadres correspondantes seront clarifiées que la qualité des prestations pourra être améliorée aux niveaux méso et micro. Les champs d'actions à ce propos font presque complètement défaut dans la stratégie et les objectifs.

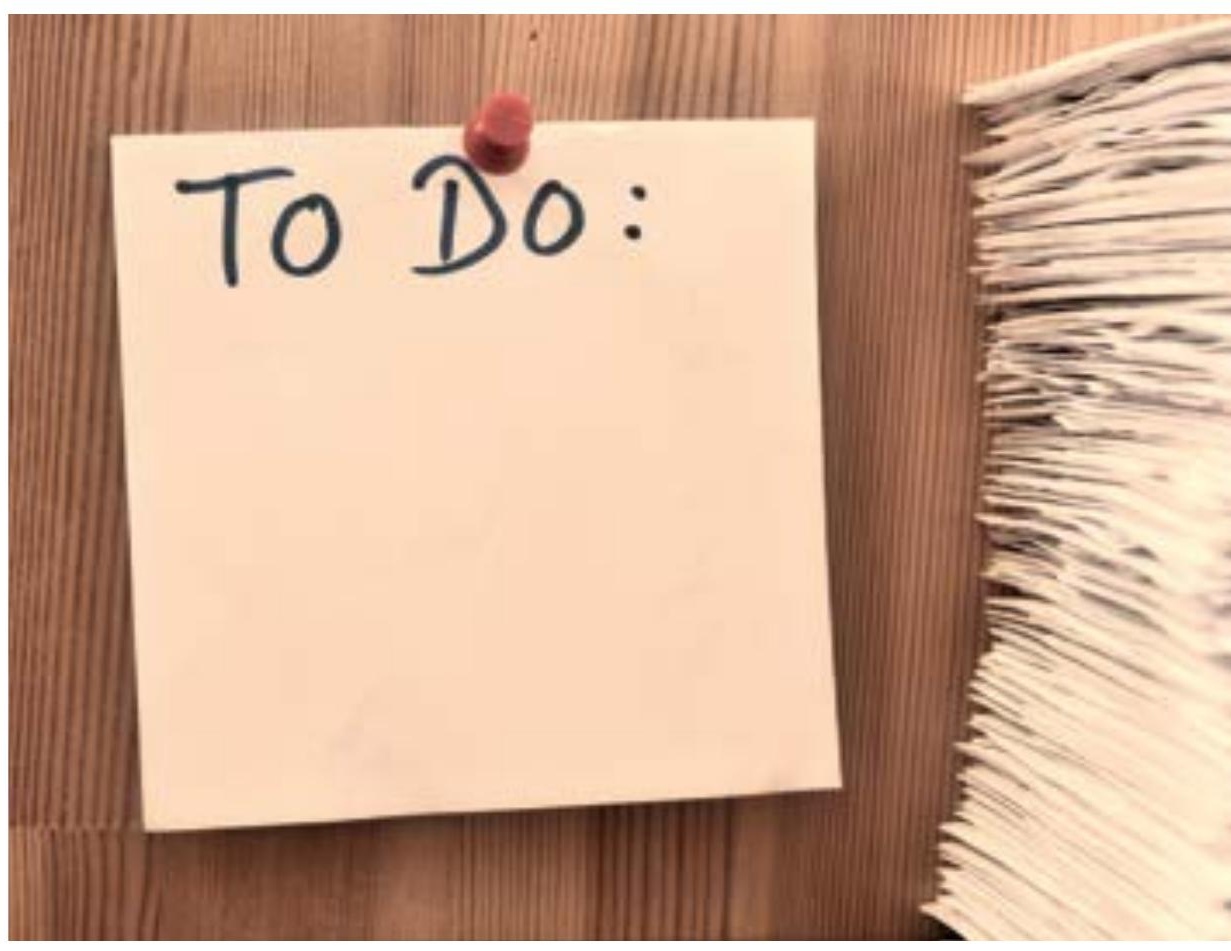




\section{Cadre temporel irréaliste}

Une mutation culturelle - mot-clé «Just Culture» - nécessite du temps et doit être accompagnée. Nous comprenons comme base de cette mutation une culture de lapprentissage et de la confiance dans laquelle le fournisseur de prestations est capable et est motivé pour mettre en ouvre les mesures de qualité également au quotidien. Ceci requiert du temps et des ressources que chaque individu doit mobiliser.

Les premiers contrats de qualité doivent être remis d'ici le $1^{\text {er }}$ avril 2022, puis autorisés et finalement mis en œuvre. Le calendrier à très court terme avec des objectifs extrêmement ambitieux et intervenant dans la liberté contractuelle des partenaires contractuels de la qualité ne peut pas être mis en œuvre dans la pratique et conduit à une règlementation excessive de l'ensemble du système de santé. Les réalités temporelles entrent ainsi complètement en collision avec les réflexions de fond de la stratégie du Conseil fédéral. Les objectifs quadriennaux doivent par conséquent être judicieusement subdivisés en objectifs à court, à moyen et à long terme.

\section{Le financement de la mise en œuvre n'a pas été clarifié}

La mise en œuvre de la stratégie mobilisera fortement tous les niveaux et exigera des ressources supplémentaires. La mise en œuvre des contrats et concepts de qualité par les associations professionnelles et les assureurs entraînent des coûts de développement et de mise en œuvre (niveau méso). Certains fournisseurs de prestations et professionnels de la santé devront introduire des systèmes supplémentaires et devront faire face à des dépenses supplémentaires pour l'exécution et la justification de leurs mesures de développement et d’assurance qualité (niveau micro). Le financement n’a pas été clarifié pour les deux. Un financement est seulement décrit grossièrement pour les travaux de développement au niveau macro. Les fournisseurs de prestations et leurs associations se voient ainsi prescrire de nombreuses tâches, sans se voir accorder le droit de participer aux débats ni des moyens financiers. Nous sommes très préoccupés par le fait que la mise en œuvre au niveau micro aux dépens de l'activité thérapeutique avec les patientes les et patients et qu’ainsi des ressources supplémen- taires soient réaffectées dans le domaine administratif. Le financement de la participation aux mesures d'assurance qualité et la répartition des coûts de développement et de mise en ouvre doivent impérativement être clarifiés avant que la mise en œuvre du contrat de qualité et le concept de développement qu'il contient ne soit mis en œuvre. Il fait sans cela craindre que la mise en œuvre échoue par absence de moyens financiers.

\section{Absence de consignes de qualité pour les assureurs}

Nous regrettons dans les présents documents l'absence de consignes de qualité pour les assureurs. Il est important que des consignes claires soient, outre les objectifs financiers également formulées à l'attention des assureurs pour la qualité de leurs prestations. Comme tant les fournisseurs de prestations que les assureurs agissent au service des patientes et des patients, les deux parties doivent pouvoir parler d'égal à égal.

\section{Habilitation de patientes et de patients et des futurs professionnels}

Du fait des éléments exposés dans la stratégie de la qualité, les patientes et les patients se verront accorder un nouveau rôle. Ils devront participer à la configuration de leurs soins de santé en toute autonomie. Cest un puissant défi à relever. Les patientes et les patients devront être aidés de façon ciblée pour être en mesure de répondre à ces exigences. Les mesures que la fédération a prévues à ce sujet afin que ceci puisse également vraiment réussir ne sont pas claires. Le rôle des organisations de patientes et de patient au niveau de la stratégie de la qualité doit en outre impérativement clarifié. Les contenus des concepts et contrats de qualités dans la formation, la formation continue et la formation postgrade des métiers de la santé doivent également être appréhendés. En parcourant la liste des adresse de consultation, nous avons été frappés de constater que les lieux de formation et de formation postgrade ne sont pas indiqués, ce que nous ne parvenons pas à comprendre.

Nous considérons que la clarification des ces questions et dautres en suspens constituent une condition préalable impérative pour une mise en œuvre réussie de la stratégie de la qualité du Conseil fédéral.

Marianne Roth est directrice de l'ASP. 